\title{
Savannah River Site Surplus Facilities Available for Reuse
}

by

R. M. Clarke

Westinghouse Savannah River Company

Savannah River Site

Aiken, South Carolina 29808

M. B. Owens

D. W. Lent

A document prepared for ADVERTISEMENT TO VENDORS

DOE Contract No. DE-AC09-89SR18035

This paper was prepared in connection with work done under the above contract number with the U.S. Department of Energy. By acceptance of this paper, the publisher and/or recipient acknowledges the U.S. Government's right to retain a nonexclusive, royalty-free license in and to any copyright covering this paper, along with the right to reproduce and to authorize others to reproduce all or part of the copyrighted paper. 


\section{DISCLAIMER}

This report was prepared as an account of work sponsored by an agency of the United States Government. Neither the United States Government nor any agency thereof, nor any of their employees, makes any warranty, express or implied, or assumes any legal liability or responsibility for the accuracy, completeness, or usefulness of any information, apparatus, product, or process disclosed, or represents that its use would not infringe privately owned rights. Reference herein to any specific commercial product, process, or service by trade name, trademark, manufacturer, or otherwise does not necessarily constitute or imply its endorsement, recommendation, or favoring by the United States Government or any agency thereof. The views and opinions of authors expressed herein do not necessarily state or reflect those of the United States Government or any agency thereof.

This report has been reproduced directly from the best available copy.

Available to DOE and DOE contractors from the Office of Scientific and Technical Information, P.O. Box 62, Oak Ridge, TN 37831; prices àvailable from (615) 576-8401.

Available to the public from the National Technical Information Service, U.S. Department of Commerce, 5285 Port Royal Road, Springfield, V.A 22161. 


\section{DISCLAIMER}

Portions of this document may be illegible in electronic image products. Images are produced from the best available original document. 


\section{Savannah River Site Surplus}

\section{Facilities Available for Reuse}

Contributors:

R. M. Clarke Rne

M. B. Owen Mbo

D. W. Lentz DWL

August 25, 1995

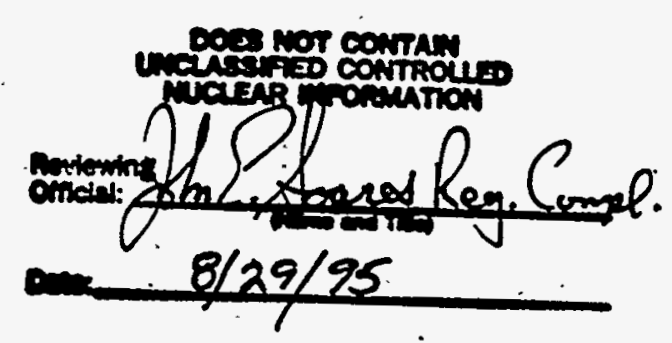

Westinghouse Savannah River Company Savannah. River Site Aiken, SC 29808 
Table of Contents:

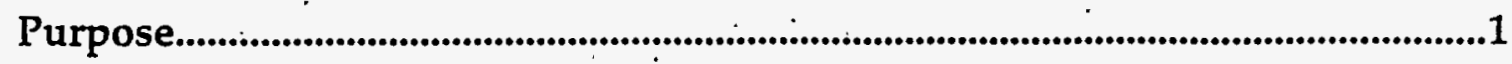

Background Information .....................................................................................1

Surplus Facility Listings ...........................................................................................................2

Major Facilities Available for Reuse ..................................................................................3

Facility Reuse Inquiry Form (from MRP 5.21)...........................................................12

Facility Reuse Contacts .12

Appendices:

A: Table of Surplus Facilities.

A.

B: Table of Non-Contaminated Surplus Facilities

C: Facility Reuse Inquiry Form 


\section{Purpose:}

The purpose of this document is to provide a current, centralized list, of Savannah River Site facilities which are surplus and available for reuse. These surplus facilities may be made available for other DOE site missions, commercial economic development reuse, or other governmental reuse. SRS procedures also require that before new construction can be approved, available surplus facilities are screened for possible reuse in lieu of the proposed new construction.

\section{Background Information:}

The Savannah River Site (SRS) was constructed during the early 1950's to produce the basic materials used in the fabrication of nuclear weapons. The Site covers 310 square miles in South Carolina. Westinghouse Savannah River Company operates the Site for the U.S. Department of Energy (DOE). With the end of the Cold War, the Site's primary mission changed from Defense Programs (nuclear materials production) to Environmental Management (waste management and environmental restoration).

A byproduct of this mission change is the availability of buildings and associated facilities that are now surplus to DOE needs. These facilities are now available for reuse by SRS organizations, other government agencies, and private industry:

When facilities are no longer needed, they are declared surplus, and are added to the Surplus Facilities Inventory Assessment (SFIA) database. When surplus, facilities are returned to use, their status is changed accordingly in the database. The SFIA database is the official SRS system for declaring a facility surplus or excess to DOE-SR needs.

Reuse of surplus facilities for new activities provides the obvious benefits of saving the user the expense and time of constructing new facilities and benefits the Department of Energy in that surveillance and maintenance costs can be eliminated and demolition costs can be deferred. There is also the 
potential for alternative arrangements leading to a facility lease or purchase of surplus installed equipment. Commercial leasing has the potential for saving DOE-SR millions of dollars annually. The WSRC Economic Development Division /Economic Development Programs is actively.pursuing onsite commercial ventures, with a primary objective of stimulating local economic development during the present period of SRS downsizing.

The.WSRC program for the management of surplus facility reuse (WSRC Manual 1B, MRP 5.21, Reise of Surplus Facilities), applies to both government and commercial ventures. The Site Facility Reuse \& Deactivation Support (SFR\&DS) section, Excess Facilities and Reactor Fuel Storage Programs Division, has the responsibility for implementing this program.

\section{Surplus Facility Listings}

Two tables of facilities are presented in the appendices. Table A lists all SRS surplus facilities (contaminated and non-contaminated). The tables are sorted by SRS area and building number. Basic information covering the facility contamination type (if any), square footage, etc. is provided.

These Tables were extracted from the current revision to the Surplus Facilities Inventory Assessment (SFIA) database. Táble B is a subset of Table $A$ and contains facilities which are not radiologically contaminated and are either clean or have minor other material contamination. Since these facilities are not radiologically contaminated, they are more readily available with little or no cleanup requirements.

WSRC management has provided special guidance for reuse of M-Area surplus facilities. The overall strategy is for WSRC to consolidate operations, where appropriate, and to relocate to other site areas as $M$ Area is deactivated and shut down. Currently; M-Area is primarily being proposed for commercial economic development. Accordingly, without senior WSRC management approval, WSRC organizations would normally not move operátions into M-Area surplus facilities. 


\section{Major Facilities Available for Reuse}

This section presents a photograph and more detailed information on major surplus facilities with the highest potential for reuse. General information covering the past facility usage, contamination (if any), and basic physical characteristics, such as the square footage is presented. More detailed information may be obtained from the personnel identified under the Facility Reuse Contacts section. 


\section{R Reactor Facility}

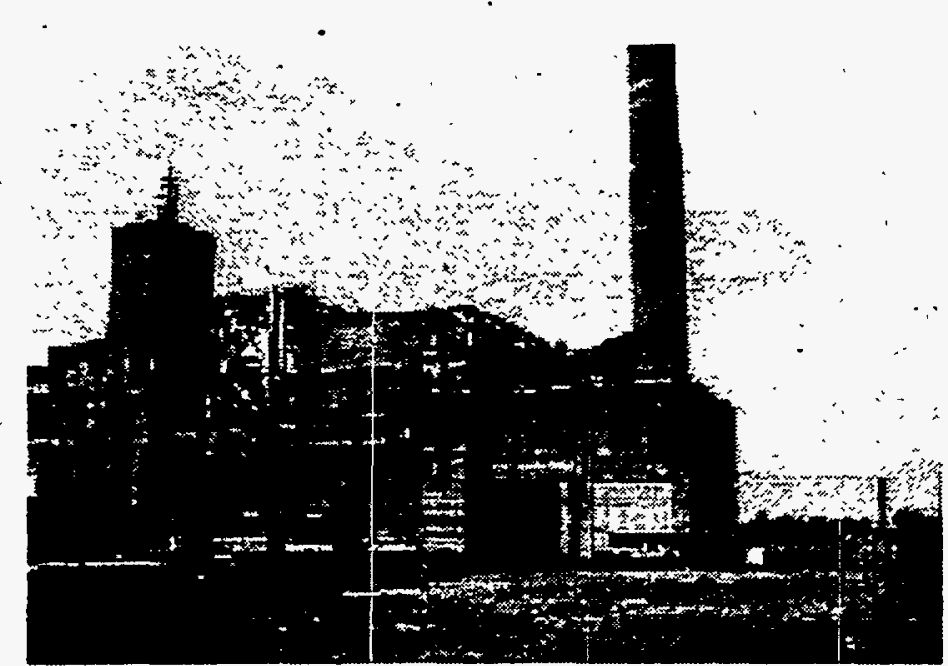

Description This large, centrally located facility was constructed between 1953 and 1956, and was shutdown in 1964. It provides ample room for manufacturing, processing or storage operations. $R$ reactor was deactivated, but has received minimum maintenance since. shutdown. The facility is built on seven floors, with approximately half a million square feet of space, mostly constructed of massive reinforced concrete. It extends from 40 feet belów grade to 150 feet above grade level. The center section of the facility containing the reactor is surrounded by the assembly area on one side arid the disassembly area on the other. The assembly area (ground level) is clean and is approximately $25,000 \mathrm{ft}^{2}$. All three sections provide large areas suitable for secure storage space. There are several support and auxiliary buildings associated with the reactor building in the area, which are gerierally not radioactively contaminated.

\section{Physical Details}

Type construction- primarily reinforced concrete construeted to be blast proof, therefore

, extremely resistant to winds and earthquakes.

\section{Physical Details}

Floor space/Vol-105-R 502,000 sq ft/ 12 million cu ft:

Contamination - Ground floor areas are predominantly clean, radioactive contaminated areas limited to reactor room, disassembly basin area and below ground process areas.

Sẹrvices- Electrical power, rail and highway access,

Low-pressure steam, potable water, sanitary sewage, fire protection are not available. 


\section{COOLING WATER BASINS}

Typical for four areas; C-Area Basin is shown, Basins also located in R, P, and L -Areas.

[Aerial view of basins shown on Reactor datasheet.]

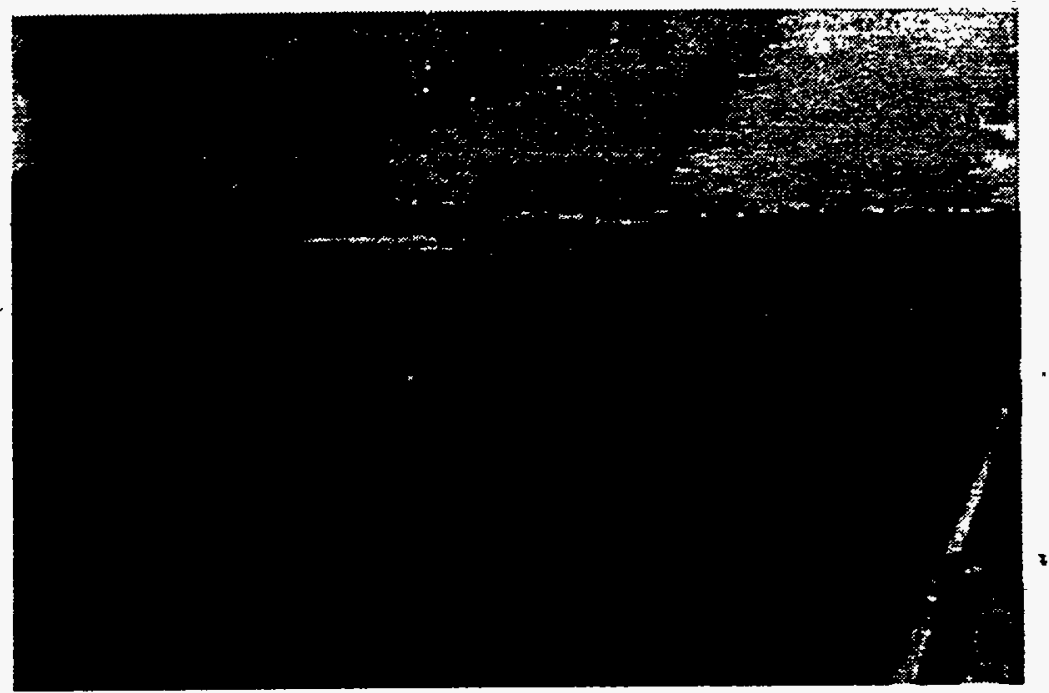

Description

These four large, open basins were constructed to collect water pumped from the Savannah River to provide cooling water for the reactors.

Initial reuse evaluations determined there was potential reuse in forms of aquaculture.

\section{Physical Details}

Type construction- reinforced concrete

Date constructed: 1952-54

Contamination: None identified; more details can be provided for each area upon request.

Large basin--24 Million gallon total capacity, subdivided into three equal sections ( $266 \mathrm{ft} \times 248 \mathrm{ft} \times 19 \mathrm{ft}$ deep) by concrete walls, sections can be isolated using existing sluice gates, sloped floor, with bottom draining capability.

Location-- R, P L \& C-Areas, are centrally located in the SRS reservation. 


\section{Alloy Fabrication Facility, 320-M}

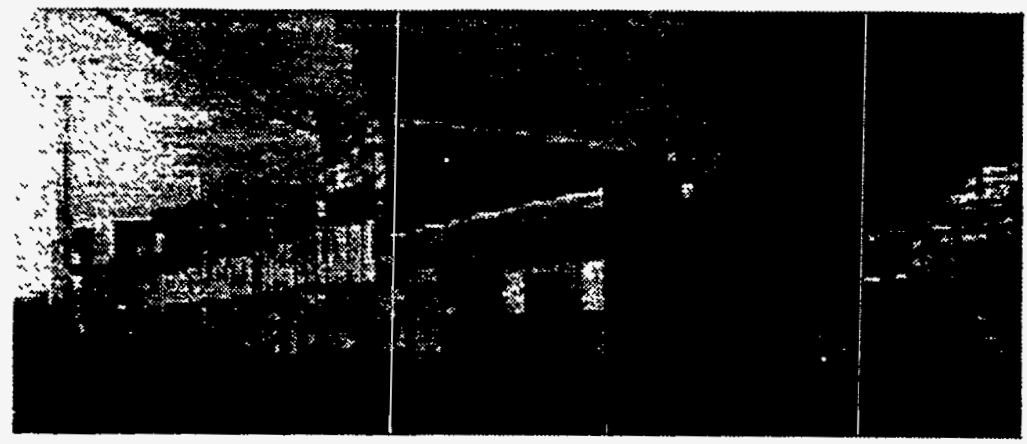

From Northeast

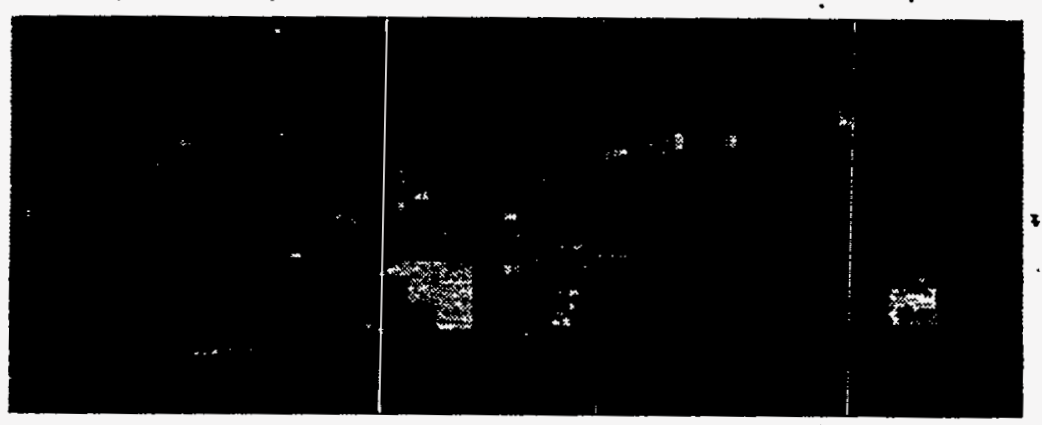

Description This, building was formerly used (1954-1993) for fabrication of reactor target tubes, control rods, and other miscellaneous components. The building contains two large production areas, 5400 and 7200 sq ft., each with bridge crane and rollup door access, a chemical cleaning area with ventilation, 2750 ton hydraulic extrusion press in the larger production area, and freight elevator to second floor area. A contiguous laboratory wing is a separate unit for reuse consideration.

Physical Details Type construction- Steel frame with Transite siding Date constructed: 1953, Floor space: 63,000 sq. ${ }^{\circ f t}$.

Contamination: Minimum localized residual contamination by depleted uranium and chemicals, and asbestos insulation.

Services- Electrical power, low-pressure steam, potable water, chilled water, instrument air, sanitary sewage, fire protection, highway access, rail access. A backup diesel-generator is also available.

Location- M-Area portion of Site Administration Area, on Site boundary. 


\section{Laboratory Facility, 320-M}

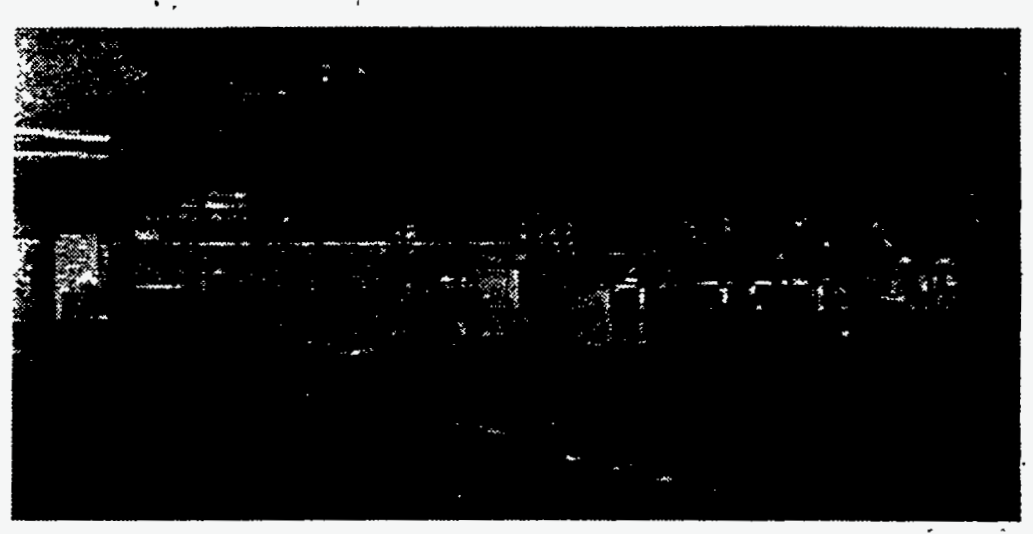

Photo 93-1073-16

\section{Description}

This facility was operated from 1953 to 1995 to support analytical needs of reactor component fabrication facilities. It contains laboratory modules with ventilation (HEPA filtration also available), offices, and a chemical storeroom. A contiguous process facility is a separate unit for reuse consideration.

\section{Physical Details}

Type construction- Steel frame with. Transite siding

Date constructed: 1953

Floor space: 13,750 sq. $\mathrm{ft}$.

Contamination: Minimum localized residual contamination by

. uranium and chemicals, asbestos insulation.

Services- Electrical power, low-pressure steam, potable water, sanitary sewage, fire protection, highway access, rail access, HEPA filtered exhaust, chilled water, plant instrument air, and backup dieselgenerator.

Location- M-Area portion of Site Administration Area, on Site boundary. 


\section{Metallurgical Laboratory Facility, 322-M}

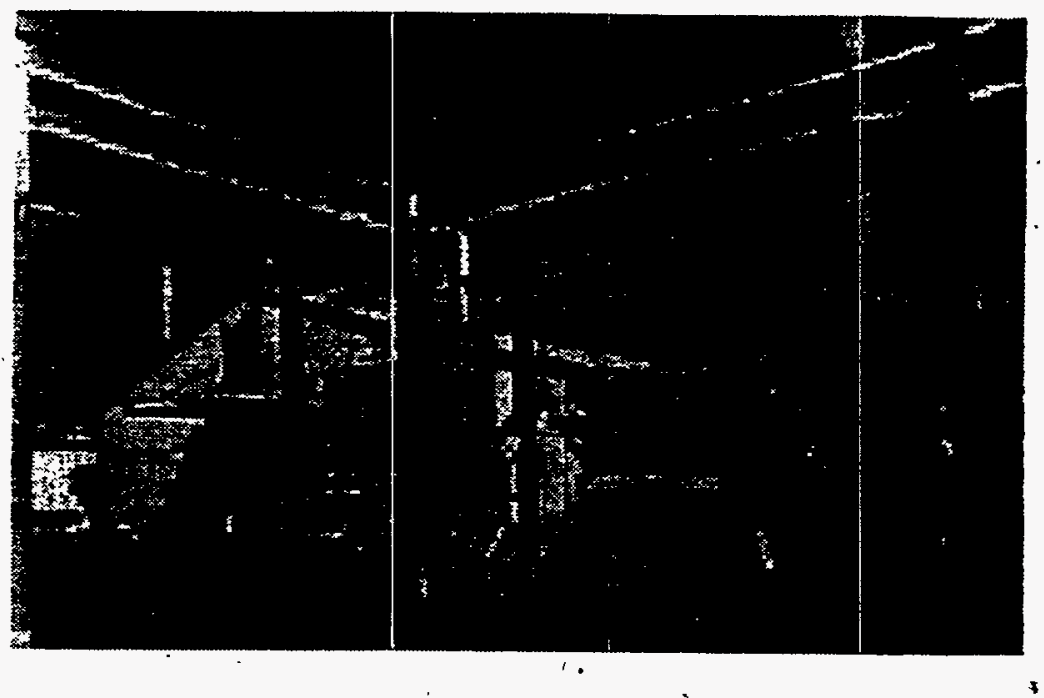

\section{Description}

This facility was formerly used from 1957 to 1995 to support reactor component fabrication facilities. It contains laboratory modules with HEPA exhaust ventilation, offices, and chemical storeroom.

\section{Physical Details}

Type construction- Wood frame with Transite siding (original).

Additions- Steel frame, with metal siding:

Date constructed: 1957, with several additions

Floor space- $11,400 \mathrm{sq} \mathrm{ft}$

Contamination - Residual uranium and chemical contamination.

Services- Electrical power, low-pressure steam, potable water; sanitary

sewage, fire protection, plant air, highway and rail access, HEPA filtered exhaust and backup diesel-generator.

Location-- Adjacent to 320-M, M-Area portion of Site Administration Area, on Site boundary. 


\section{Target Slug Fabrication Facility, 313-M •}

\section{Description}

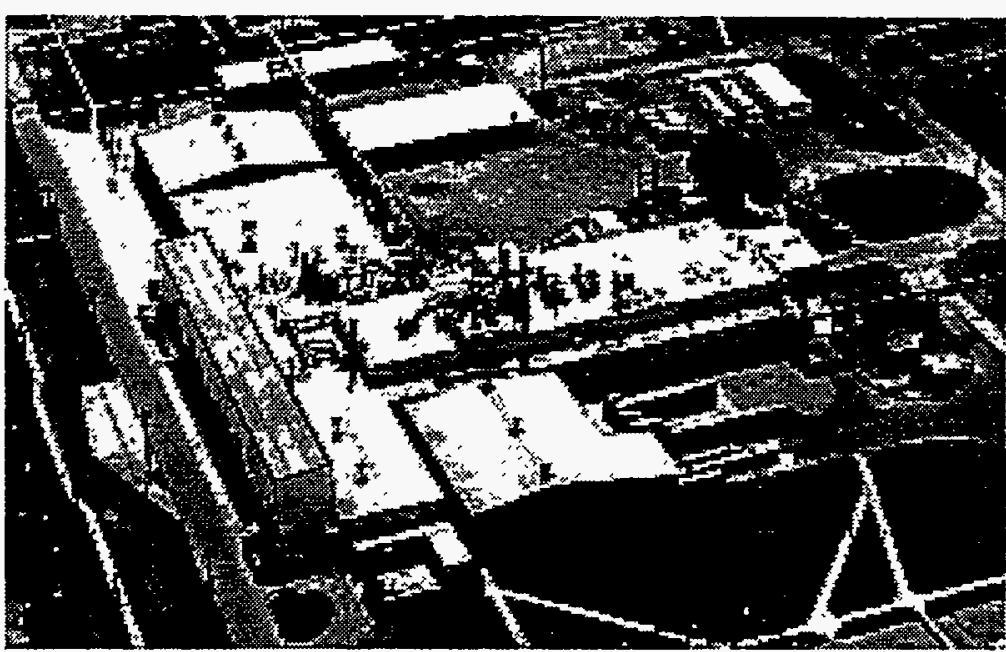

This facility operated from 1954 to 1992 for fabrication of depleted uranium slugs used as targets in the reactors. It contains a large production area, rollup door access, loading dock, autoclave pit, a chemical cleaning area with ventilation, slug canning equipment, some specialized metalworking equipment in the large production area, tool storage and warehouse areas, maintenance shops, and office space. This facility has contiguous liquid chemical waste collection, handling and processing equipment.

\section{Physical Details}

Type construction- Steel frame with Transite siding

Date constructed: 1952, with additions ..

Total Floor space: 76,000 sq. $\mathrm{ft}$.

Contamination: Residual contamination by depleted uranium and chemicals, asbestos insulation.

Services- Electrical power, low-pressure steam, potable water, plant air, sanitary sewage, fire protection, highway access, rail access, and backup diesel-generator.

Location-- M-Area portion of Site Administration Area, on Site boundary.

Condition-- Minimum repairs required for roof and fire protection system. 


\section{- Naval Fuels Manufacturing Facility, 247-F}

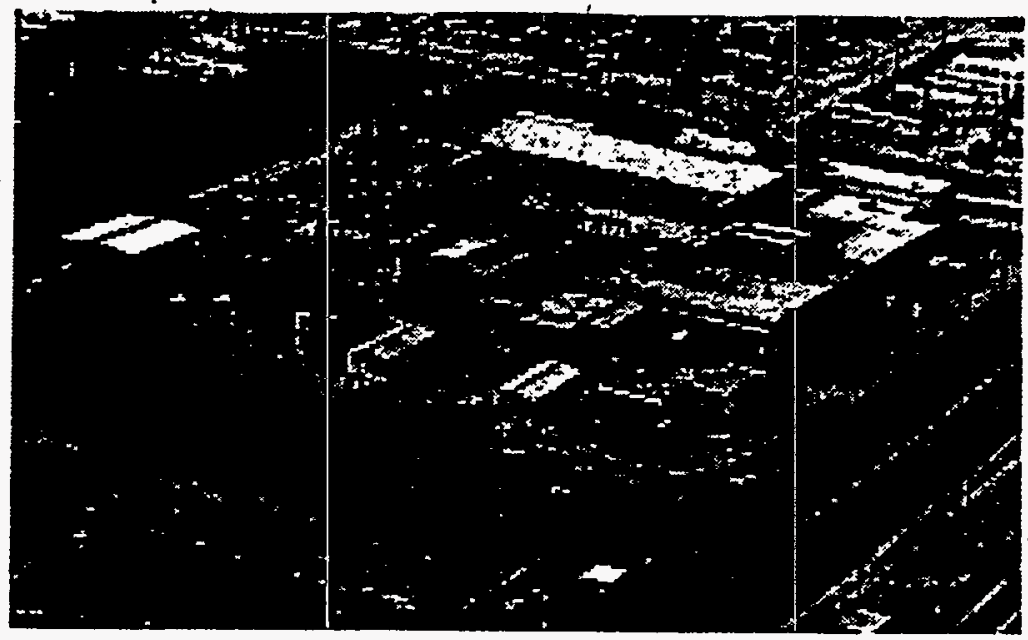

\section{Description}

The Naval Fuels Manufacturing Facility was constructed between 1983 and 1985. It is a structural steel frame building on concrete slab floors. The facility was. in the process of startup when it was decided to permanently shut it down. The facility contains a central process core with radiological contamination, a clean vault, a radiologically contaminated laboratory, a warehouse/receiving area, four maintenence shop areas, a, computer room, waste processing area, and a two story clean administration wing. The process core is a major portion of the facility. The facility is surrounded by several support buildings.

\section{Physical Details}

Type construction- Steel frame with prefabricated insulated exterior walls

Dạte constructed: Mid 1980s.

Floor space: 95,000 sq. $\mathrm{ft}$.

Contamination: Some areas are radiologically contaminated; there is no asbestos containing material

Services- Electrical power, potable water, sanitary sewage, fire protection, highway access .

Location- F-Area. 


\title{
Construction Craft Buildings- (717-8P and 717-9L)
}

\author{
No photo available.
}

\section{Desscription}

The construction craft buildings were built to house construction craftsman, parts and tool storage, administration support personinel and engineers working on the reactor restart program. The buildings. are typical prefab metal buildings using steel framed construction with metal siding and roof. The buildings are insulated and each have $a$ number of package $H \& V$ units servicing the building located at. ground level around the building and at least one large vehicle door. The buildings have adequate power supply and potable water supply. The inside of the buildings were completed with two levels of wood frame construction to provide offices, parts and tool storage, break areas, etc. This wood construction can not remain in the building since it does not meet NFPA codes. However, if removed, the building could serve as a large conditioned warehouse, shop, or other production related function.

\section{Physical Details}

Type of Construction-Prefabricated steel framed metal building. with vinyl backed insulation in the walls and ceiling.

Date Constructed: January 1989.

Floor Space: 7000 sq. ft. (L-area), 7486 sq. ft. (P-area)

Contamination: No radiological or environmental contamination suspected. No asbestos containing materials.

Services: Electriçal power, potable ẉater, sanitàry sewer tap available, $\mathrm{H} \& \mathrm{~V}$.

Lơcation: Buildings located in L-area and P-areas.

Other Information: Located in unrestricted security area for personnel. No TLD's required but must monitor coming out of area. 


\section{Facility Reuse Inquiry Form}

A sample of the-Facility Reuse Inquiry form (taken from WSRC Manual 1B, MRP 5.21) is included in Appendix $C$, and is to be completed when there is a project or a potential need for a facility. The completed form will specify the special requirements for an individual facility. These requirements could be anything from air conditioned storage space, an area suitable for office space or some special cranes or equipment to support the new project. Once received by the Site Facility Reuse \& Deactivation (SFR\&D) section, the facility requirements will be utilized to attempt to locate a suitable facility which meets the specifications. Tours of prospective buildings and negotiations will then be arranged as required. In the overall process, the SFR\&D section serves as a broker to match up surplus facilities with potential facility users. Please refer to WSRC Manual 1B, MRP 5.21 when completing the form, and direct any questions to the appropriate contact listed below.

\section{Facility Reuse Contacts}

There are several contacts for inquiring about facility reuse. The appropriate initial contact is based upon on-site or off-site usage as indicated below:

\section{On-site contact:}

Randy Clarke, 707-C, 557-9879 or

Marley Bruns, 707-C, 557-9468.

Off-site commercial or Government users contact:

Cris. W. Van Horn;

U. S. Department of Energy

Savannah River Operations Office

P. O. Box A, Building 703-46A

Aiken, S. 29802 .

(803) $725-53.13$

or

Marc V. Eidson, Manager, Economic Development Programs,

Westinghouse Savannah River Company

P. O. Box A, Building 999-W

Aiken, S. C. 29802

(803) $652-1850$ 


\begin{tabular}{|c|c|c|c|c|c|c|c|c|c|c|}
\hline \multirow[t]{2}{*}{ Area } & \multirow{2}{*}{$\frac{\text { Building No. }}{\ldots}$} & \multirow[t]{2}{*}{ Facillty Name } & \multirow[t]{2}{*}{ Status } & \multirow{2}{*}{ Year } & \multicolumn{2}{|c|}{ Contaminated } & \multirow{2}{*}{$\begin{array}{l}\text { Resp: } \\
\text { Div. }\end{array}$} & \multirow{2}{*}{ Construction Type } & \multicolumn{2}{|r|}{ Floors. } \\
\hline & & & & & Rad? & Other & & & No. & Space (ft2) \\
\hline A. & $607-001 \mathrm{~A}$ & SEWAGE TREATMENT PLANT, 607-001A & Abandone & 1993 & $N$ & S & SS & Steel Frame & 1 & 3300 \\
\hline $\overrightarrow{\mathbf{C}}$ & $108-001 \mathrm{C}$ & EMERGENCY DIESEL ROOM, 108-1C & Shutdown & 1995 & $N$ & $\bar{s}$ & $\bar{F}$ & Reinforced Concrete & 2 & 5000 \\
\hline C. & $108-002 \mathrm{C}$ & EMERGENCY DIESEL ROOM, 108-2C. & Shutdown & 1995 & $N$ & S. & $\mathbf{F D}$ & Reinforced Concrete & 2 & 5000 \\
\hline $\overrightarrow{\mathbf{C}}$ & $1.86-000 \mathrm{C}$ & COOLING WATER BASIN (25M GALLONS), 186-C & Shutdown & 1995 & $\underline{\mathbf{I}}$ & $\ddot{s}$ & FD. & Reinforced Concrete & 1) & 200600 \\
\hline$\overline{\mathbf{C}}$ & $190-000 \mathrm{C}$ & BOOSTER PUMP BUILDING, 191-C & Shutdown & 1995 & $\bar{N}$ & $\bar{T}$ & $\overline{\mathbf{P D}}$ & Reinforced, Concrete & 2 & 520 \\
\hline $\mathbf{C}$ & $608-000 \mathrm{C}$ & CHANGE FACILITY, 608-C & Shutdown & 1995 & N. & $\overline{\mathbf{T}}$ & FD. & Steel Frame & $i$ & 96 \\
\hline $\overrightarrow{\mathbf{C}}$ & $6+4-002 C$ & EFFLUENT MONITOAING BUILDING, 614-2C. & Shutdown & $1 \overline{9} 95$ & s & $\mathbf{T}$ & FD & Steel Frame & 1 & 180 \\
\hline C & $701-001 \mathrm{C}$ & AREA GATEHOUSE, 701-1C & Shutdown & 1995 & $N$ & $T$ & PD. & Reinforced Concrete & 1 & 2500 \\
\hline $\mathbf{C}$ & $701-002 C$ & EXCLUSION AREA FENCE ENTRY POINT, 701-2C & Shutdown & 1995 & N & $\dot{\mathbf{T}}$ & $\overline{F D}$ & Concrete/Mason Block & 1 & 1680 \\
\hline $\mathbf{C}$ & $701-004 C$ & SHELTER FOR SECURTTY EQUIPMENT, 701-4C & Shutdown & 1995 & $\ddot{\mathbf{N}}$ & $\ddot{T}$ & $\mathbf{F D}$ & Steel Frame & i) & $60 \overline{0}$ \\
\hline$\underline{\mathbf{C}}$ & $701-006 \mathrm{C}$ & GUARDHOUSE, $701-6 C$ & Shutdown & 1995 & $\bar{N}$ & $\ddot{T}$ & $\mathbf{F}$ & Other. & 1 & 40 \\
\hline $\mathbf{C}$ & $717-000 \mathrm{C}$ & HOT SHOPISTORAGE SHED, $717-\mathrm{C}$ & Shutdown & $19 \overline{95}$ & $\bar{Y}$ & $\mathbf{T}$ & $\overline{\mathbf{F D}}$ & Reinforced Concrete & il & 1000 \\
\hline C & $904-001 \mathrm{C}$ & COOLING WATER EFFLUENT SUMP, 904-1C & Shutdown & 1995 & $\underline{Y}$ & $T$ & FD & Reinforced Concrete & 1 & 375 \\
\hline $\bar{c}$ & $904-89 \mathrm{GI}$ & RETENTION BASIN TANKV100-C CONT,,904-89G-1 & Shutdown & 1995 & $\bar{Y}$ & $\dot{\underline{T}}$ & $\overrightarrow{F D}$ & Other & N/A & $500 k$ gal. \\
\hline $\mathrm{D}$ & $401-000 D$ & GAS PLANT, 401-D & Shutdown & 1995 & 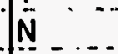 & 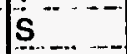 & FD & Reinforced Concrete & 1 & 1440 \\
\hline D. & $401-001 \mathrm{D}$ & GAS PLANT CONTROL BUILDING, 401-1D & Shutdown & 1995 & N & s & PD & Steel Frame .... & i) & 700 \\
\hline $\mathbf{D}$ & $402-000 \mathrm{D}$ & HEAVY WATER TANK FARM; 402-D & Shutdown & 1995 & $N$ & $\mathbf{s}$ & $\overrightarrow{F D}$ & Reinforced Concrete & i & 11600 \\
\hline D. & $412-001 \mathrm{D}$ & HEAVY WATER EXTRIACTION TOWERS \& PIPING & Shutdown & 1995 & $\mathbf{N}$ & $\underline{Y}$ & FD & Aeinforced Concrete & 1 & 27000 \\
\hline$\ddot{\mathbf{D}}$ & $412-004 D$ & MASK MAINTENANCE BUILDING, 412-4D & Shutdown & 1995 & N & $\bar{s}$ & FD & Reinforced Concrete & 1 & 1350 \\
\hline $\bar{D}$ & $412-005 \mathrm{D}$ & SHELTER AND SHOP BUILDING, 412-5D & Shutdown & 1995 & $\bar{N}$ & $\underline{\mathbf{s}}$ & $\overline{\mathbf{F D}}$ & Reinforced Concrete & 1 & 1200 \\
\hline$\underline{\mathbf{D}}$ & $412-008 \mathrm{D}$ & TUBE BUNDLE INSPECTION, 412-8D & Shutdown & 1995 & $N$ & $\bar{N}$ & FD. & Reinforced Concrete & i & 450 \\
\hline $\mathbf{D}$ & $412-009 \mathrm{D}$ & BOLT STORAGE, 412-9D & Shutdown & 1995 & $N$ & $\mathbf{I}$ & $\mathbf{F D}$ & Reinforced Concrete & il & 1200 \\
\hline$\underline{D}$ & $412-010 \mathrm{D}$ & LUBE BUNDLE CLEANSS'NG SHELTER, 412-10D. & Shutdown & 1995 & $N$ & $s$ & FD & Reintorced Concrete & 1 & 700 \\
\hline $\bar{D}$ & $419-000 D$ & FLAAE TOWER, 419-D & Shutdown & 1995 & $\bar{N}$ & $\underline{s}$ & PD & Reinforced Concrete & 1 & $250^{\circ} 0$ \\
\hline$D$ & $421-005 \mathrm{D}$ & LOADING DOCK, 421-5D & Shutdown & 1995 & $N$ & $\bar{s}$ & $F$ & Roinlorced Concrele & 1 & 200 \\
\hline$F$ & $211-004 \mathrm{~F}$ & SAMPLINGHOUSE, 211-4F & Abandone & 1993 & $\underline{Y}$ & $\underline{Y}$ & $\overline{N M}$ & Steel Frame & 1 & 100 \\
\hline$F$ & $235-000 \mathrm{~F}$ & METALUAGICAL BUILDING, 235-F (PUFF) & Shutdown & 1993 & $\bar{Y}$ & $y$ & $\mathrm{NM}$ & Reinforced Concrete & 2 & 7015 \\
\hline $\mathbf{F}$ & $235-000 \mathrm{~F}$ & METALLURGICAL BUILDING, 235-F (PEFF) & Shutdown & 19993 & Y & $Y$ & NM & Reinforced Concrete & 2 & 1000 \\
\hline $\mathbf{F}$ & $235.000 \mathrm{~F}$ & METALLURGICAL BUILDING, 235-F (OLDMET LAB) & Shutdown & $19 \underline{9} 3$ & $\ddot{Y}$ & $y$ & MM & Reinforced Concrete & 1 & 435 \\
\hline $\mathbf{F}$ & $247-000 \mathrm{~F}$ & NAVAL FUELS MAN.BLDG.247-F,(COAEL & Shutdown & 1993 & $\frac{Y}{Y}$ & r & 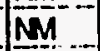 & Concrete/Mason Block & $\dot{2}$ & 34726 \\
\hline $\mathbf{F}$ & $247-007 F$ & ECPROCESSBUILDING, 247-7F & Other & 1993 & $\overrightarrow{\mathbf{Y}}$ & $\dot{Y}$ & $M$ & Steel Frame & 5 & 5760 \\
\hline $\mathbf{F}$ & $247-011 \mathrm{~F}$ & OUTSIDE COLDFEED STORAGE, 247-11F & Shutdown & 1993 & $N$ & $\ddot{y}$ & $\mathrm{NM}$ & Steel Frame & 1. & 194 \\
\hline $\mathbf{F}$ & $247-012 \mathrm{~F}$ & OUTSIDE COLD FEED STORAGE, 247-12F & Shutdown & 1993 & $N$ & $\bar{Y}$ & $N M$ & Steel Frame & 1 & 3520 \\
\hline
\end{tabular}

Key: N - No, Y - Yes, T - Suspect No S - Suspect Yes 


\begin{tabular}{|c|c|c|c|c|c|c|c|c|c|c|}
\hline \multirow[t]{2}{*}{ Area. } & \multirow{2}{*}{ Bullding No. } & \multirow{2}{*}{ Facillity Namo } & \multirow[t]{2}{*}{ Status. } & \multirow{2}{*}{ Year } & \multicolumn{2}{|c|}{ Contaminated } & \multirow{2}{*}{$\begin{array}{l}\text { Resp. } \\
\text { Div. }\end{array}$} & \multirow[t]{2}{*}{ Construction. Type } & \multicolumn{2}{|r|}{ Floors } \\
\hline & & & & & Rad? & Other & & & No. & Space (ft2) \\
\hline $\mathbf{F}$ & $254-002 F^{\circ}$ & DIESEL GENERATOR FACILITY, 254-2F & Shutdown & 1994 & $\mathbf{N}$ & $Y$ & $\mathbf{N M}=$ & er & 2 & 600 \\
\hline $\boldsymbol{F}$ & $284-000 F$ & POWERHOUSE, $2844-000 \mathrm{~F}$ & Abandone & 1993 & $\mathbf{N}$ & $\vec{N}$ & ss & Reiniorced Concrete & 3 & 30000 \\
\hline $\mathbf{F}$ & $607-001 \mathrm{~F}$ & SEWAGE TAEATMENT PLANT/DIGESTER, 607-001F & Abandone & 1993 & $N$ & $\mathbf{N}$ & SS & Reinforced Concrete. & 1 & 2475 \\
\hline$\overline{\mathbf{G}}$ & $681-006 \mathrm{G}$ & PAR POND PUMP HOUSE, 681-006G & down & 1993 & $\underline{\mathbf{N}}$ & N & ss: & & 1 & 4800 \\
\hline$\underline{\mathrm{H}}$ & $24.1-916 \mathrm{H}$ & WST STRG TK(FRMRLY 241000H TK16), 241-916H & Shutdown & 1993 & $\underline{\mathbf{Y}}$ & 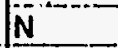 & $\underline{H L}$ & Other & $N / A$ & i.03MM gal. \\
\hline$\dot{H}$ & $242-003 H$ & ATE PUMP TANK PIT, 242-3H & Shutdown & 1993 & $\overline{\mathbf{Y}}$ & $N$ & HL & Reinforced Concrete & 1 & 400 \\
\hline K. & $105-001 \mathrm{~K}$ & BASIN DEIONIZER PAD, 105-1K & indby & & $\bar{Y}$ & $T$ & FD & & 1 & 2000 \\
\hline $\mathbf{K}$ & $105-0.13 K$ & HEAVY WATER STORAGE FACILITY, 105-13K & dby & & $\underline{\mathbf{S}}$ & $S$ & FD & & 1 & 2500 \\
\hline $\mathbf{K}$ & $185-$ & HOUSE), 185-K & tdown & & $\bar{N}$ & $\underline{N}$ & 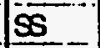 & & 12 & $6 \overline{50} 0$ \\
\hline$\underline{K}$ & $185-001 K$ & CHLORINA & tdown & 1993 & $\mathrm{~N}$ & $\mathrm{~N}$ & Ss & & i) & $\overline{6000}$ \\
\hline$\overline{\mathbf{K}}$ & $904-088 G$ & RETENTION BAS & & & $\underline{s}$ & $\bar{Y}$ & FD & 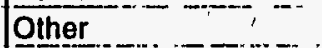 & $N / A^{\circ}$ & $1.8 \mathrm{MM}$ \\
\hline$L$ & $\overline{105-0}$ & SAND FIL & own & & $\bar{Y}$ & $\bar{s}$ & $\overline{\mathbf{F D}}$ & ncrete & 1 & 900 \\
\hline$L$ & $106-$ & EEDWTER STORAGE TANK & awn & & $\underline{v}$ & $S$ & & crete & 1 & 640 \\
\hline$\underline{L}$ & $107-c$ & COOLING & wn & & $\underline{\mathbf{s}}$ & $\mathrm{T}$ & & icrete. & i) & 1000 \\
\hline$\underline{L}$ & $108-$ & EMERGEN & own & 19 & $\bar{N}$ & $\bar{Y}$ & $\mathrm{FD}$ & crete & 2 & 5110 \\
\hline$\underline{L}$ & 108- & EMERGEN & down & 1995 & $\bar{N}$ & S & $\overline{F D}$ & crete. & 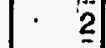 & 5000 \\
\hline$\underline{L}$ & $L$ & $E \mathrm{~L}, 108-4 \mathrm{~L}$ & wn & & $N$ & $\mathrm{~s}$ & PD & & i & 320 \\
\hline$L$ & $109-c$ & 109-L & own & 19 & $\mathbf{s}$ & $\underline{s}$ & $\mathbf{P D}$ & crete & 1 & 305 \\
\hline$L$ & $\overline{D L}$ & $0-\mathrm{L}$ & own & & $N$ & $\underline{\mathbf{s}}$ & $\overline{\mathrm{AD}}$ & crete. & 1 & 240 \\
\hline$L$ & & $152.1 \mathrm{~L}$ & $\overline{w n}$ & & $\bar{N}$ & $\bar{T}$ & $\overline{\mathbf{F D}}$ & grete & 1 & $17 i 6$ \\
\hline$L$ & $152-\mathrm{C}$ & OR $704-1,2 \& 3-L, 152-4 L$ & own & & $\underline{N}$ & $\mathbf{T}$ & & crete & i) & 50 \\
\hline- & 152-c & $2-10 \mathrm{~L}$ & & & $N$ & $T$ & & grete & 1 & 190 \\
\hline 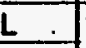 & $\mathbf{L}$ & GAL & Nn & 18 & $T$ & $T$ & FD & rete & N/A & $25 \mathrm{MM}$ gal. \\
\hline- & & $91-L$ & wn & & $\mathbf{N}$ & $T$ & & erete & $2]$ & 440 \\
\hline$\underline{L}$ & & & & & $\bar{N}$ & $\mathrm{~T}$ & & & i. & 150 \\
\hline L & $2 \mathrm{~L}$ & LDING, 614-2L & $\overline{w n}$ & 19 & $T$ & $T$ & $\overline{F D}$ & & $1)$ & 270 \\
\hline$\underline{L}$ & $1 L$ & $L$ & own & & $\bar{N}$ & $\bar{N}$ & & & i) & 800 \\
\hline L & & JHAGE BUILDING, & & & $N$ & $\underline{s}$ & $\mathbf{F D}$ & & 1 & 972 \\
\hline$\underline{L}$ & $715-000 L$ & GASOLINE SERVICE STATION-715L & Shutdown & 1995 & $N$ & S: & $\mathbf{F}$ & Reinforced Concrete & i. & \\
\hline 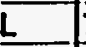 & $717-009 \mathrm{~L}$ & CRAFT BUILI & wn & & $\mathbf{T}$ & $\underline{\mathbf{S}}$ & $\mathbf{F D}$ & $\operatorname{cod} \mathrm{C}$ & 2 & 7000 \\
\hline 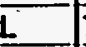 & 723-002L & CHANGE BUIL & & 19 & $\overrightarrow{\mathbf{S}}$ & & FD & & i) & 108 \\
\hline & $723-003 \mathrm{~L}$ & CHANGE BUILDING, 723-3L & Standby & $19 \overline{95}$ & $\mathbf{S}$ & $T$ & E्E & Steel Fi & & 108 \\
\hline & $312-000 \mathrm{M}$ & TANK FARM, 312-M (two 5000 gallon tanks) & Shutdown & 1992 & $\mathbf{N}$ & $\mathbf{Y}$ & E & Other & $\bar{N} / \mathrm{A}$ & 1280 \\
\hline
\end{tabular}

Key: N-No, $Y$ - Yes,

$T$ - Suspect No

S - Suspect Yes
Table of Surplus Facilities 


\begin{tabular}{|c|c|c|c|c|c|c|c|c|c|c|}
\hline \multirow[t]{2}{*}{ Area } & \multirow[t]{2}{*}{ Bullding No. } & \multirow[t]{2}{*}{ Facility Name } & \multirow[t]{2}{*}{ Status } & \multirow{2}{*}{$\begin{array}{l}\text { Year } \\
\text { Surplus }\end{array}$} & \multicolumn{2}{|c|}{ Contaminated } & \multirow{2}{*}{$\begin{array}{l}\text { Resp. } \\
\text { Div. }\end{array}$} & \multirow[t]{2}{*}{ Construction Typo } & \multicolumn{2}{|r|}{ Floors } \\
\hline & & & & & Rad? & Other & & & No. & Space- (ft2) \\
\hline$M$ & $313-0$ & ZANSFER FACILITY, 313-M,CTF & wn & & $Y$ & $\mathbf{Y}$ & E & & 2 & 6800 \\
\hline $\bar{M}$ & $\bar{M}$ & उER, 318 & vate & & & $\mathrm{Y}$ & 垔 & Reinforced Concrete & 1 & 512 \\
\hline$M$ & $\underline{O M}$ & ALLOY FABRICATION FACILITY, 320-M & hutdown & 1993 & $\underline{Y}$ & $\underline{Y}$ & $\underline{\underline{E}}$ & Steel Frame. & 2 & 63288 \\
\hline$M$ & $\vec{M}$ & LLABORATORY, 320-M & & $19 \overline{95}$ & & $\mathbf{Y}$ & 正 & Steel Frame & 1 & 13752 \\
\hline $\bar{M}$ & $\overline{4}$ & BUILDING, $320-7 M$ & own & 1995 & $\bar{N}$ & iN & 百 & Ōther & 1 & 80 \\
\hline$M$ & $\underline{M}$ & METALLURGICAL LABO & own & $1 \overline{9}$ & $\underline{\underline{Y}}$ & $\bar{Y}$ & 互 & Steel \& Wood Frame & 1 & 11411 \\
\hline $\bar{M}$ & $322-0$ & SUPPLY SHED, 322-1M & wn & 1995 & $\underline{N}$ & $\bar{N}$ & 画 & Other & 1 & $i 68$ \\
\hline$M$ & & $313 M$ & $2 w n$ & & $\underline{Y}$ & $Y$ & & rele/Mason Block & 3 & 75912 \\
\hline P & & (ATEA), 105-13P & pwn & & $\bar{r}$ & $\underline{Y}$ & FD. & nncrete & 1 & 1930 \\
\hline P & 1 & STORAGE TANK. & own & 1 & $\dot{\underline{Y}}$ & $\ddot{Y}$ & $\overline{D D}$ & ncrete & i) & 600 \\
\hline $\mathbf{P}$ & & EIR, 107-P & wn & & $\bar{s}$ & $\ddot{s}$ & $\overline{\mathbf{P D}}$ & crele & 1 & 864 \\
\hline $\bar{P}$ & & $8-1 P$ & own & & $N$ & $\mathrm{~s}$ & $\overline{\mathbf{F D}}$ & icrete & 1 & 5110 \\
\hline$P$ & & 8-2P. & own & & $N$ & $\underline{S}$ & FD. & crete & 2 & 5000 \\
\hline$\vec{P}$ & & $\overline{K 109}$ & own & & $\underline{s}$ & $\bar{Y}$ & FD & crete & 1 & \\
\hline$\ddot{P}$ & 110 & $P$ & own & & $\bar{N}$ & $\underline{T}$ & $\ddot{F D}$ & oncrete & 1 & 240 \\
\hline$\ddot{P}$ & 184 & COAL CAI & own & 19 & $\mathbf{N}$ & $\underline{\mathbf{N}}$ & ss & Concrete & 1 & 352 \\
\hline $\mathbf{P}$ & 1 & & own & 1 & $T$ & $T$ & $\overline{\mathrm{FD}}$ & icrete & 2 & 440 \\
\hline P. & 60 & CHANGE FACI & own & & $T$ & $T$ & FD & & 1 & 10 \\
\hline p & & EFFLUENT MO & own & & $N$ & $\tilde{T}$ & $\overline{\mathrm{D}}$ & Fel Frame & 1 & 144 \\
\hline P & 70 & $Y, 701-4 P$ & Shutdown & is & $\bar{N}$ & & FD. & Other & 1 & 200 \\
\hline $\bar{P}$ & $7 \overline{1}$ & & own & & $\ddot{N}$ & $\mathbf{N}$ & $\mathrm{FD}$ & Steel & 1 & 900 \\
\hline $\mathrm{P}$ & & & & & $\bar{N}$ & $\ddot{T}$ & $\bar{n}$ & & i) & 900 \\
\hline$P$ & $P$ & & Nn & & $N$ & $N$ & & & 1 & 100 \\
\hline $\overrightarrow{\mathbf{P}}$ & 717 & 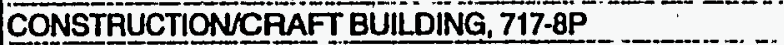 & own & & $N$ & $\bar{N}$ & $\overline{\mathrm{FD}}$ & inforced Concrete & 2 & 186 \\
\hline$P$ & & & & 19 & $\bar{T}$ & $\mathrm{~T}$ & FD & Reinforced Concrete & $r^{\cdot} \quad 1$. & 1250 \\
\hline $\bar{P}$ & 9 & $4086-1$ & $\mathrm{Nn}$ & 1 & $\bar{s}$ & $\underline{s}$ & FD & Other & IN/A & 500K gal. \\
\hline R & & & own & & $\bar{Y}$ & $\bar{Y}$ & & Concrete & 7 & 1000000 \\
\hline R & & SIORAGE I & & 1995 & $\underline{Y}$ & $\underline{s}$ & & Concrete & 1 & 200 \\
\hline A & & WEIR, 107-R & Shutdown & 1995 & $\bar{N}$ & $\sqrt{s}$ & $\overline{\mathbf{F D}}$ & Reinforced Concrete & 1 & 1012 \\
\hline $\mathrm{R}$ & & OM $108-18$ & own & 1995 & $\bar{N}$ & $\vec{s}$ & $\overline{\mathbf{E D}}$ & Reinforced Concrete & 2 & 5110 \\
\hline R & $2 \mathrm{R}$ & 요-2금 & wn & & $\overline{\mathbf{N}}$ & s & FD & Concrete & 2 & 5110 \\
\hline$R$ & & $\bar{N}$ K, 109-R & own & & $\ddot{Y}$ & $s$ & $\overline{F D}$ & d Concrete & 1 & .140 \\
\hline R & $151-002 R$ & Electrical Distribution Building & Shutdown & 1995 & $\mathbf{N}$ & $\mathbf{N}$ & Ss & Reinforced Concrete & 2 & 13500 \\
\hline
\end{tabular}

Key: N-No, Y - Yes,

$T$ - Suspect No

S - Suspect Yes 


\begin{tabular}{|c|c|c|c|c|c|c|c|c|c|c|}
\hline Area & Bullding No. & Facllity Name. & Status & Year & Con & minated & Resp. & Construction Type & & Floors \\
\hline & ' & 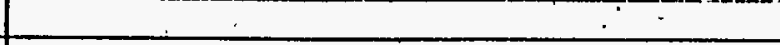 & & Surplus & Rad? & Other & Div. & & No: & Space (tt2) \\
\hline$\underline{\mathbf{R}}$ & $183-001 \mathrm{~A}$ & WATER CLARIFICATION FAC. STORAGE BASIN, 183-1R & Shutdown & 1995 & $\mathbf{N}$ & $\underline{S}$ & $\mathbf{P D}^{\circ}$ & Reinforced Concrète & 1 & . 102000 \\
\hline $\mathrm{R}$ & 183-002A & WATER CLARIFICATION FAC. STORAGE BASIN, 183-2A & Shutdown & $19 \overline{95}$ & $\bar{N}$ & $s$ & FD & Reinforced Concrete & & 6000 \\
\hline R & $186-000 \mathrm{R}$ & COOLING WATER BASIN (25 M GALLONS), 186-R & Shutdown & 1995 & $\mathbf{N}$ & $\underline{S}$ & $\mathbf{P D}$ & Reinforced Concrete & 1 & 200000 \\
\hline $\mathbf{R}$ & $190-000 R$ & COOLNG WATER PUMP HOUSE, 190-R & Shutdown & 1995 & $\underline{N}$ & $\underline{S}$ & FD & Reinforced Concrete & i & 10000 \\
\hline $\mathbf{R}$ & 191:000R & STANDBY PUMP HOUSE, 191-A & Shutdown & 1995 & $\bar{N}$ & $\mathbf{S}$ & FD & Reinforced Concrete & 1 & 370 \\
\hline R & $704-000 R$ & ADMINISTRATION BUILDING, 704-R & Shütown & 1995 & $\bar{Y}$ & $\bar{Y}$ & $\overline{\mathbf{F D}}$ & Reinforced Concrele & 1 & 15000 \\
\hline
\end{tabular}


sOᄉ loadsns - S

oN loedsns - $\perp$.

'sal - $\lambda$, ON - N : Kay

\begin{tabular}{|c|c|c|c|c|c|c|c|c|c|c|}
\hline 0009 & 2 & 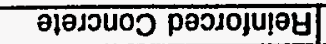 & $\boldsymbol{\alpha}$ & $\mathbf{S}$ & $\mathbf{N}$ & 966! & UMOpInUS & 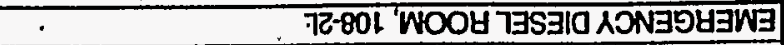 & $7200-801$ & \\
\hline$\overline{019}$ & $\bar{z}$ & 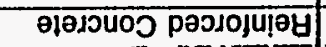 & at & $\lambda$ & $N$ & 9661 & ümopinus & 7L-80I ' & $7100-80 !$ & \\
\hline 509 & $\ddot{i}$ & गөपाO & $\overline{s s}$ & $\ddot{N}$ & N. & $866 i$ & üм̄opinùs & Y्र1-S81 उSกÖHषOL & 시으-98i & $\ddot{\ddot{y}}$ \\
\hline$\overline{9} \overline{9}$ & $z$ & गबपा० & $\overline{s s}$ & N. & $\bar{N}$ & $\bar{\varepsilon} \overline{6} \mathbf{6 i}$ & ümopinús & 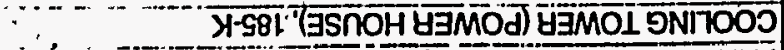 & $2000-981$ & $\ddot{\underline{y}}$ \\
\hline $00 \ddot{8}$ & 1 & ग्पाO & $\bar{s}$ & $\bar{N}$ & $\mathbf{N}$ & E661 & umopinus & 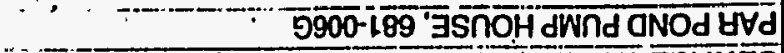 & $5900-189$ & 9 \\
\hline$s \angle b \ddot{z}$ & $i$ & 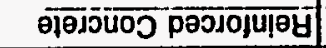 & ss & $\mathrm{N}$ & $\bar{N}$ & $\overrightarrow{\mathrm{E} 6 \mathrm{t}}$ & èuopuequ & 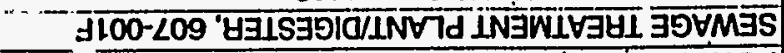 & $\exists 100-\angle 00$ & $\exists$ \\
\hline $0000 \varepsilon$ & $\varepsilon$ & 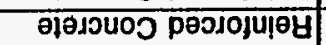 & SS & $\mathbf{N}$ & $\mathbf{N}$ & E661 & euopurqy & $\pm 000-p 8 C^{\prime} \exists S n O H \mathrm{H} \exists M O d$ & $\pm 000-682$ & $\exists$ \\
\hline $00 \overline{9}$ & $i$ & ग्पा० & $\overline{W N}$ & ㄱ. & N- & 666 & ümopinǘs & JE+69E & $\overline{\mathbf{2} 00}-t \overline{\mathrm{g}} \overline{\mathrm{z}}$ & تص \\
\hline б̄̄s̄ & $i$ & 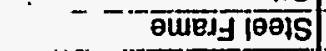 & $\bar{W}$ & A & Ni & $\overline{\varepsilon 6} \overline{6}$ & umopinus & 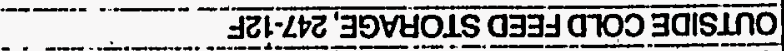 & $0-\angle t z$ & $\underline{j}$ \\
\hline 61 & 1 & өuredy & WN & 시 & $\ddot{N}$ & $866 i$ & üMopinus & 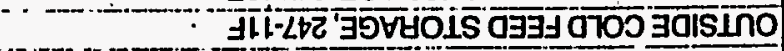 & [O- & $\underline{J}$ \\
\hline $00 \bar{z}$ & $i$ & 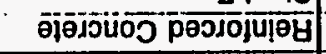 & a) & $\mathbf{s}$ & $\mathrm{N}$ & 9661 & ümopinus & 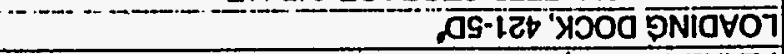 & $\overline{0 S 00}-i \overline{z t}$ & $\overline{\mathbf{a}}$ \\
\hline $0 \overline{0 S Z}$ & I & 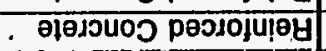 & $\mathbf{a}$ & $\mathbf{S}$ & $N$ & 9661 & umopinus & Q-616' $\mathrm{A} \exists M O \perp \exists \mathrm{BV7 \textrm {H }}$ & $0-616$ & $\overline{\mathbf{a}}$ \\
\hline $00<$ & $i$ & 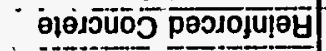 & ä & s. & - & 9661 & uḾpinus & 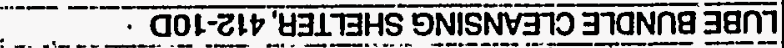 & $\overline{0} 01 \overline{0}-\bar{z} 1 \bar{b}$ & a \\
\hline O0Z1 & i & 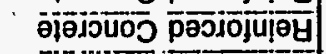 & a्t & 1 & Nit. & $966 i$ & ümopinus & 06-216 3984015 170 & a600-2it & $\bar{a}$ \\
\hline$\dot{s i}$ & 1 & 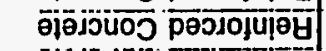 & $\overline{\mathbf{a}}$ & N & $\overline{\mathrm{N}}$ & $\overline{\mathbf{s}} \overline{6} \mathbf{2}$ & ümópinus & 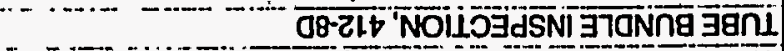 & $\overline{0}-\vec{z} i \vec{b}$ & $\bar{a}$ \\
\hline 0021 & $i$ & 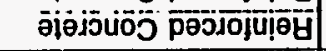 & $\overline{\mathbf{a}}$ & si & N & $966 i$ & umopinus & 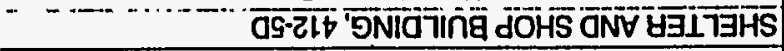 & $\overline{50}-\tilde{z}+\ddot{\nabla}$ & $\ddot{\mathrm{a}}$ \\
\hline OSE I & $\bar{l}$ & 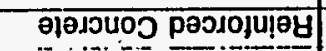 & a) & $\mathbf{s}$. & N & 966! & UMopinus & 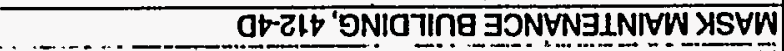 & $0-\bar{Z} L t$ & $\overline{\mathbf{G}}$ \\
\hline $00 \angle Z$ & 1 & 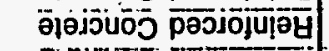 & $\overline{\mathbf{a}}$ & 시 & N] & 966i & umopinus & פNIdId 8 SUEMO & $\overline{0}-\dot{z} i \dot{i}$ & $\ddot{a}$ \\
\hline $0091 i$ & $i$ & 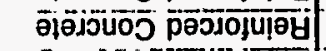 & ät & $\overline{\mathbf{s}}$ & N] & $\underline{\mathbf{s}} \mathbf{6} \underline{\underline{ }}$ & uMOpinus & 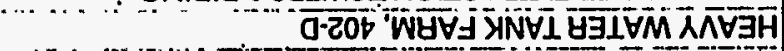 & $\bar{\alpha} \mathbf{0} \overline{0} \overline{0}-\bar{c} \overline{0} \bar{c}$ & $\underline{a}$ \\
\hline $00<$ & 1 & 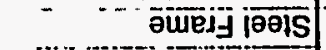 & $\overline{\mathbf{a}}$ & $\ddot{\mathbf{s}}$ & in & $966 i$ & ümópinus & 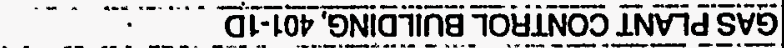 & âtoo-ıot & $\overrightarrow{\mathbf{a}}$ \\
\hline $0 \bullet \dot{t}$ & $i$ & 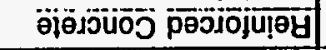 & $\overline{\mathbf{a}}$ & $\overline{\mathbf{s}}$ & $\mathrm{N}$ & S66i & ùMōiñüs: & Q-10b'INVTd SVI & $0000-i \overline{00}$ & $\overline{\mathbf{a}}$ \\
\hline $0 t$ & I & 10410 & $\mathbf{a}$ & & $N$ & 9661 & UMopinus & 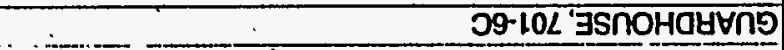 & $30-102$ & 2 \\
\hline 009 & t. & 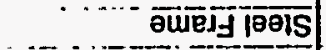 & a) & & $\mathrm{N}$ & $966 i$ & UMopinus & OV-LOL'INヨWdIINO & $\overline{00-1} \overline{0}$ & 30 \\
\hline $08 \ddot{9}$ & $i$ & 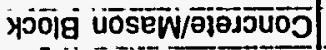 & a & i) & Ni & $\mathbf{s} \mathbf{6} \overline{6}$ & ūMōpinus & 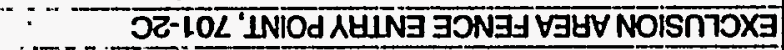 & $0200-102$ & $\overline{0}$ \\
\hline $00 \bar{s} \bar{z}$ & $\mathrm{i}$ & 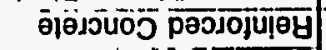 & (स) & & $\mathbf{N}$ & $\underline{s} 6 \overline{6} \underline{1}$ & ümopiñus & 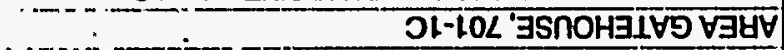 & $0-10<$ & $\underline{3}$ \\
\hline$\overline{9} \overline{6}$ & $i$ & 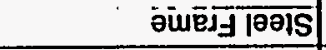 & $\overline{\mathbf{a}}$ & I) & $\mathrm{N}$ & $\overline{9} \overline{6} \overline{6}$ & ùMopiñús & 0.809 XITIOVJ $95 \mathrm{NVHO}$ & $0000-\overline{809}$ & 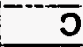 \\
\hline$\overline{029}$ & $\bar{z}$ & 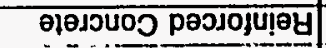 & a्) & 1 & $\mathbf{N}$ & 966! & uMopınus & 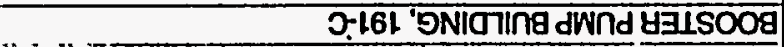 & $0000-061$ & $\overline{0}$ \\
\hline$\overline{0} \overline{0} \overline{9} \overline{0} 0 \ddot{z}$ & l & 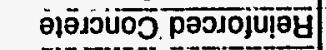 & $\underline{\mathbf{a}}$ & $\bar{s}$ & I & $\underline{\mathbf{9}} \underline{\underline{6}} \underline{i}$ & 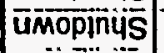 & 0.981 '(SNOTTVפWSZ) NIS & $0000-98 i$ & 5 \\
\hline $000 ̈ \mathbf{s}$ & $z$ & 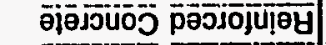 & $\overline{\mathbf{a}}$ & & $\bar{N}$ & $\bar{s} \overline{6} \overline{6} \overline{1}$ & uмiōpinüs & 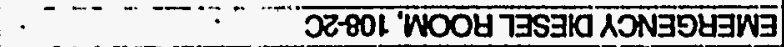 & $2200-\overline{80} i$ & $\overline{3}$ \\
\hline 0009 & 2 & 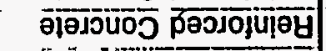 & ä) & $\overline{\mathbf{s}}$ & N & $\overline{\mathbf{s}} \overline{\mathbf{6}} \mathbf{i}$ & üмöpınus & OL-80 'WOOY TES & $\overline{0100}-\overline{80} \overline{\mathrm{I}}$ & 5 \\
\hline $00 \ddot{\varepsilon} \bar{\varepsilon}$ & 1 & 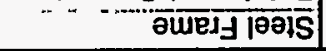 & $\overline{\mathbf{S S}}$ & $-\bar{s}$ & $\cdots \bar{N}$ & $\bar{\varepsilon} 6 \overline{61}$ & ēंōp̈uëqü & $\forall 100-\angle 09 \perp N \forall / \perp N \exists W \perp \forall \exists \forall \perp \exists 5 \forall M \exists S$ & $\forall 100-\angle 09$ & $\bar{\forall}$ \\
\hline (दu) oveds & ON & \multirow[b]{2}{*}{ 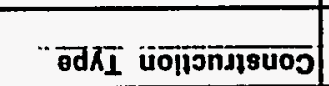 } & ताव & 10410 & ¿pert & snidins & & & & \\
\hline 810019 & & & - d808 & \multicolumn{2}{|c|}{ 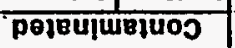 } & $180 \hat{\lambda}$ & snjeis & . OUIEN KIIIISE & ON bulp\|n & $\bar{\theta} \bar{V}$ \\
\hline (zIH) ejeds & 0 & & $\cdot n \mid 0$ & 104io & 2pey & snjdins & & & & \\
\hline S.100|J & & อdKI uolionnisuog & नdsou & \multicolumn{2}{|c|}{ 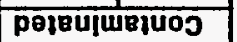 } & $\operatorname{se\theta } \lambda$ & 8njeis & 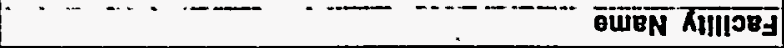 & ON bulp|lng & E्गय \\
\hline
\end{tabular}




\begin{tabular}{|c|c|c|c|c|c|c|c|c|c|c|}
\hline \multirow[t]{2}{*}{ Area } & \multirow{2}{*}{ Bullding No. } & \multirow{2}{*}{ Facillity Name $\quad \ldots \ldots \ldots$} & \multirow[t]{2}{*}{ Status } & \multirow{2}{*}{ Year } & \multicolumn{2}{|c|}{ Contaminated } & \multirow{2}{*}{ Divesp: } & \multirow[t]{2}{*}{ Construclion Type } & \multicolumn{2}{|r|}{ Floors } \\
\hline & & & & & Rad?. & OOther & & & No. & Space (1t2) \\
\hline $\mathbf{L}$ & 108-004L. & 903 EXHAUST FANEMERGENCY DIESEL, 108-4L & Shutdown & 1995 & $\mathbf{N}$ & $S$ & FD & Reinforced Concrete & 1 & 320 \\
\hline$\underline{L}$ & $110-000 \mathrm{~L}$ & HELIUM STORAGE TANKS, $110 \mathrm{~L}$ & Shutdown & 1995 & $\bar{N}$ & $\mathrm{~s}$ & $\overline{\mathbf{P D}}$ & Reinforced Concrete & 1 & 240 \\
\hline LL. & $15 \underline{2-0} 0 \underline{1} \underline{L}$ & CONTAINMENT SUBSTATION, 152-1L & Shutdown & 1995 & $\underline{N}$ & $T$ & FD & Reinforced Concrete & i) & 1716 \\
\hline L & $152-004 L$ & SECONDARY SUBSTATION FOA 704-1,2\& 3-L, 152-4L & Shutdown & 1995 & $\bar{N}$ & $T$ & PDD & Reinforced Concrete & 1 & 50 \\
\hline$\underline{L}$ & $152-010 \mathrm{~L}$ & TRANSFORMEA $300 \mathrm{KVA}, 152-10 \mathrm{~L}$ & Shutdown & $1 \overline{995}$ & N. & $\underline{T}$ & $\underline{\mathbf{F}}$ & Reinforced Concrete & 1 & 190 \\
\hline$\underline{L}$ & $186-000 \mathrm{~L}$ & COOLING WATER BASIN (25M GALLON), 186-000L & Shutdown & 1995 & $\mathbf{I}$ & I. & $\overline{\underline{P}}$ & Reinforced Concrete & $N / A$ & $25 \mathrm{MM}$ gal. \\
\hline $\mathbf{L}$ & $191.000 \mathrm{~L}$ & BOOSTER PUMP BUILDING, 191-L & Shutdown & 1995 & $\overline{\mathbf{N}}$ & $\bar{T}$. & $\overline{\mathbf{F D}}$ & Reinforced Concrete & 2 & 440 \\
\hline$\underline{L}$ & $501-000 \mathrm{~L}$ & OLD SECURITY DIESEL, 501-L. & Shutdown & 1995 & $\mathbf{N}$ & $T$ & $\mathbf{F D}$ & Reinforced Concrete & 1 & $1 \underline{50}$ \\
\hline$\underline{L}$ & $614-002 \mathrm{~L}$ & EFFLUENT MONITORING BUILDING, 614-2L & Shuidown & $\overrightarrow{1995}$ & $\mathbf{T}$ & $\mathrm{T}$ & $\underline{\mathbf{F D}}$ & Steel Frame & i) & 270 \\
\hline$\underline{L}$ & $709-001 \mathrm{~L}$ & FIRE TRUCK SHED, 709-1L & Shutdown & 1995 & $\mathbf{N}$ & $\underline{N}$ & $\overline{\mathbf{F D}}$ & Steel Frame & i) & 800 \\
\hline L & 711-000L & MAINTENANCE MATL. STORAGE BUILDING, 711-L & Shutdown & 1995 & $\mathbf{N}$ & & $\overline{\mathbf{R D}}$ & Steel Frame & $!$ & 972 \\
\hline $\mathbf{L}$ & $715-000 \mathrm{~L}$ & GASOLINE SERVICE STATION-715L & Shutdown & 1995 & $\bar{N}$ & $\bar{s}$ & $\overline{\mathbf{F D}}$ & Reinforced Concrete & i. & 45 \\
\hline L & $717-009 L$ & CRAFT BUILDING, L-AREA & Shutdown & 1995 & $T$ & $\underline{s}$ & FD & Aeinforced Concrete & 2 & 7000 \\
\hline$\underline{M}$ & $312-000 \mathrm{M}$ & TANK FAFM, 312-M & Shutdown & 1993 & $\bar{N}$ & $\underline{Y}$ & E一. & Other & $N / A$ & 12000 \\
\hline$\underline{M}$ & $320.007 M$ & COMPAESSED GAS STORAGE BUILDING, $320-7 \mathrm{M}$ & Shutdown & 1995 & $\mathbf{N}$ & $\bar{N}$ & 互 & Other & 1 & 80 \\
\hline $\mathbf{M}$ & $322-001 \mathrm{M}$ & SUPPLY SHED, 322-1M & Shutdown & 1995 & $\mathbf{N}$ & $N$ & 正 & Öther & i. & 168 \\
\hline $\mathbf{P}$ & $108-001 \mathrm{P}$ & EMERGENCY DIESEL ROOM, 108-1P & Shutdown & $19 \overline{5}$ & $\mathbf{N}$ & $S$ & $\overline{\mathbf{F D}}$ & Feinforced Concrete & i. & 5110 \\
\hline $\mathbf{P}$ & 108-002P. & EMERGENCY DIESEL ROOM 108-2P & Shutdown & 1995 & $\mathbf{N}$ & s & $\underline{F D}$ & Heinforced Concrete. & 2 & 5000 \\
\hline$\underline{P}$ & $110-000 \mathrm{P}$ & HELIUM STORAGE TANKS, 110-P & Shutdown & 1995 & $\mathbf{N}$ & I & $\mathrm{FD}$ & Reinforced Concrete & $\overline{1}$ & 240 \\
\hline $\mathbf{P}$ & 184-003P & COAL CAR SHAKER, 184-3P & Shutdown & 1993 & $N$ & $\underline{\mathbf{N}}$ & $\underline{\mathbf{S}}$ & Feinforced Concrete & 1 & 352 \\
\hline $\mathbf{P}$ & 191-00,0P & BOOSTER PUMP HOUSE, 191-P & Shutdown & 1995 & $\mathbf{T}$ & $\underline{T}$ & FD & Reinforced Concrete & 2 & 440 \\
\hline $\mathbf{P}$ & $608-000 \mathrm{P}$ & CHANGE FACILITY, 608-P & Shutdown & 1995 & $\mathbf{T}$ & $\bar{T}$ & $\mathbf{F D}$ & Wood & 1 & $5 \ddot{310}$ \\
\hline $\mathbf{P}$ & 614-002P & EFFLUENT MONITORINGGUILDING, 614-2P & Shutdown & 1995 & $\mathbf{N}$ & $\mathbf{T}$ & $\mathbf{F D}$ & Steel Frame & $!$ & 144 \\
\hline $\mathbf{P}$ & 701-004P & HP MONITORING FACILITY, 701-4P. & Shutdown & 1995 & $N$ & 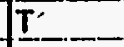 & FD. & Other & 1. & 200 \\
\hline $\mathbf{P}$ & $711-000 P$ & MAINTENANCE MATERIAL STORAGE BLDG., 711-P & Shutdown & 1.995 & $\mathbf{N}$ & $N$ & FD & Steel Frame & 1 & 900 \\
\hline $\mathbf{P}$ & $711-001 P$ & STORAGE BUILDING, 711-1P & Shutdown & 1995 & $\underline{\mathbf{N}}$ & $\mathrm{T}$ & $\mathbf{F D}$ & Steel Frame & 1 & $\underline{9} 00$ \\
\hline $\mathbf{P}$ & $715-000 \mathrm{P}$ & GASOLINE STATION, 715-P & Shutdown & 1995 & $\bar{N}$ & $\bar{N}$ & $\overline{\mathbf{F D}}$ & Other & 1 & 100 \\
\hline $\mathbf{P}$ & 717-008P & CONSTRUCTIONCRAFT BUILDING, 717-8P & Shutdown & 1995 & $\underline{N}$ & $\underline{\mathbf{N}}$ & $\mathbf{F D}$ & Peinforced Concrete & 2 & 7486 \\
\hline $\mathbf{P}$ & $717-009 P$ & PIPE FAB SHED, 717-9P & Shutdown & 1995 & $\bar{T}$ & $\mathrm{~T}$ & $\mathbf{F D}$ & Reinforced Concrete & $1)$ & 1250 \\
\hline$\underline{\boldsymbol{R}}$ & 107-000R & COOLING WATEREFFLUENT WEIR, 107-R & Shutdown & 1995 & $\mathbf{N}$ & $\underline{\mathbf{s}}$ & $\mathbf{F D}$ & Reinforced Concrete & 1 & 1512 \\
\hline$\underline{\mathbf{R}}$ & $108-001 \mathrm{R}$ & EMERGENCY DIESEL ROOM, 108-1R & Shutdown & 1995 & $\underline{N}$ & $\underline{s}$ & $\underline{\mathbf{F}}$ & Reinforced Concrete & 2 & 5110 \\
\hline $\overrightarrow{\mathbf{R}}$ & 108-002R & EMERGENCY DIESEL ROOM, 108-2R & Shutdown & 1995 & $N$ & $\mathbf{S}$ & $\mathbf{F D}$ & Reinforced Concrete & 2 & 5110 \\
\hline $\mathbf{R}$ & 151-002R & Electrical Distribution Building & Shutdown & 1995 & $\mathbf{N}$ & $\mathbf{N}$ & $\mathbf{s s}$ & Reinforced Concrete & 2 & 13500 \\
\hline
\end{tabular}

Key: N - No, Y - Yes,

$T$ - Suspect No

S - Suspect Yes
Table of Clean and Non-Radiologically Contaminated Surplus Facilities 


\begin{tabular}{|c|c|c|c|c|c|c|c|c|c|c|}
\hline \multirow{2}{*}{ Area } & \multirow{2}{*}{ Building No. } & \multirow{2}{*}{ Facillity Namo } & \multirow{2}{*}{ Status. } & \multirow{2}{*}{ Year } & \multicolumn{2}{|c|}{ Contaminated } & \multirow{2}{*}{$\begin{array}{l}\text { Resp. } \\
\text { Div. }\end{array}$} & \multirow[t]{2}{*}{ Construction Type } & \multicolumn{2}{|r|}{ Floors } \\
\hline & & & & & Rad? & Other & & & No. & Space (ft2) \\
\hline $\mathbf{R}$ & $183-0018$ & WATER CLARIFICATION FAC. STORAGE BASIN, 183-1R & Shutdown & 1995 & N & $\underline{\mathbf{s}}$. & $\underline{F}$ & Reinforced Concrete & 1 & 102000 \\
\hline $\mathbf{R}$ & $183-002 \mathrm{R}$ & WATER CLARIFICATION FAC. STORAGE BASSIN, 183-2R & Shutdown & $1 \overline{995}$ & $\bar{N}$ & $\bar{s}$ & $\overline{\mathbf{F D}}$ & Reinforced Concrete & 1 & 6000 \\
\hline$\underline{R}$ & $186-000$ A & COOLING WATER BASIN (25 M GALLONS), 186-R & Shutdown & $19 \underline{9} 5$ & $\mathbf{N}$ & $\underline{\mathbf{S}}$ & PD. & Reinforced Concrete. & 1 & 200000 \\
\hline $\mathbf{R}$ & $190-000 \mathrm{~A}$ & COOLING WATEA PUMP HOUSE, 190-R & Shutdown & $19 \underline{9} 5$ & & $\underline{\mathbf{s}}$ & FD & Reinforced Concrete & 1 & 10000 \\
\hline R & $191-000 \mathrm{R}$ & STANDBY PUMP HOUSE, 191-A & Shutdown & $1 \overline{995}$ & $\bar{N}$ & $\bar{s}$ & $\overline{\mathrm{FD}}$ & Peinforced Concrete & 1 & 370 \\
\hline
\end{tabular}


EFR-TDD-950060

SRS Surplus Facilities Available for Reuse

August 25, 1995

Facility Reuse Inquiry

(Return Completed Form to Manager, FTSTD\&D)

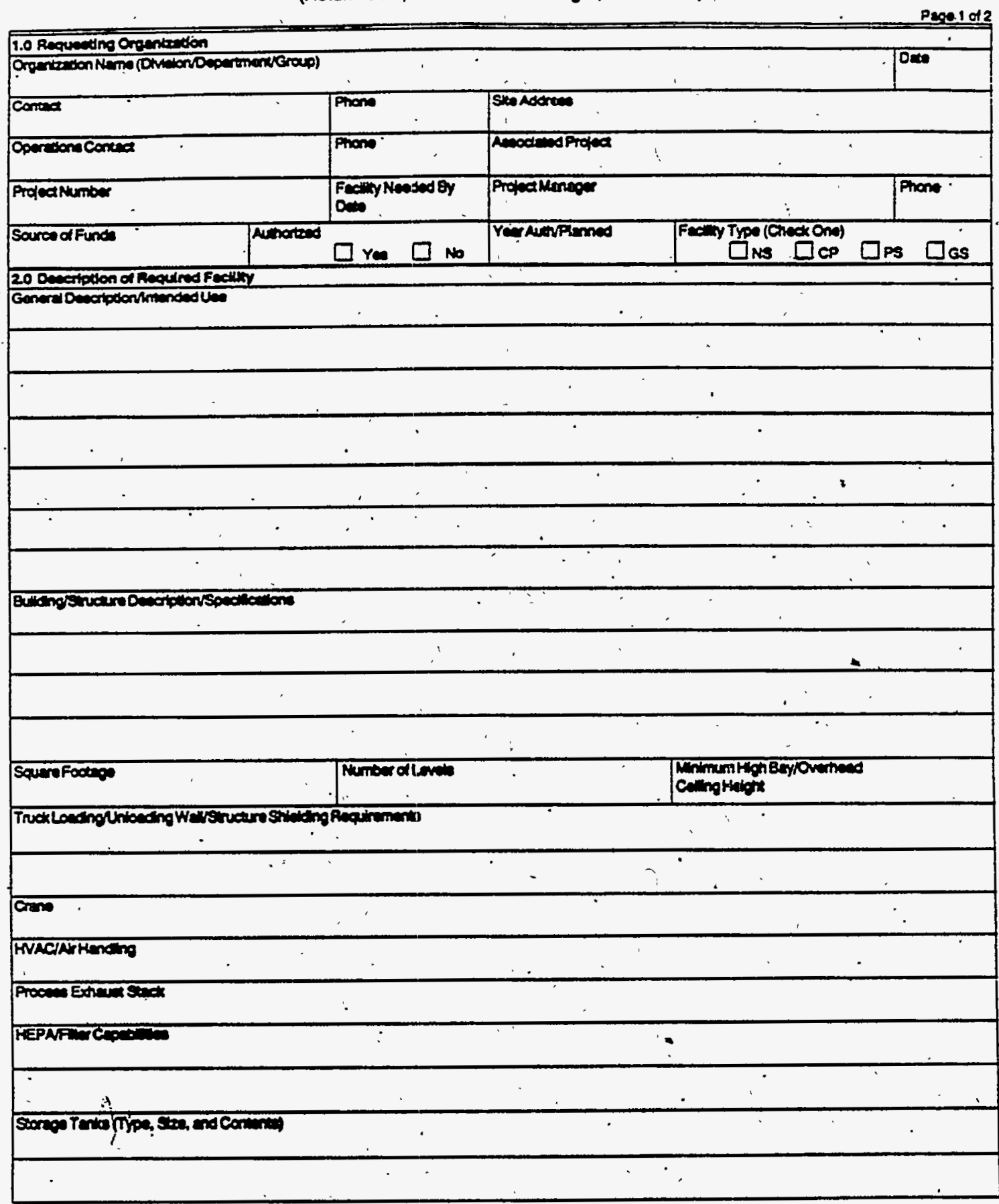

\section{Appendix C}

Facility Reuse Inquiry Form 
Fàcility Reuse Inquiry (ctd)

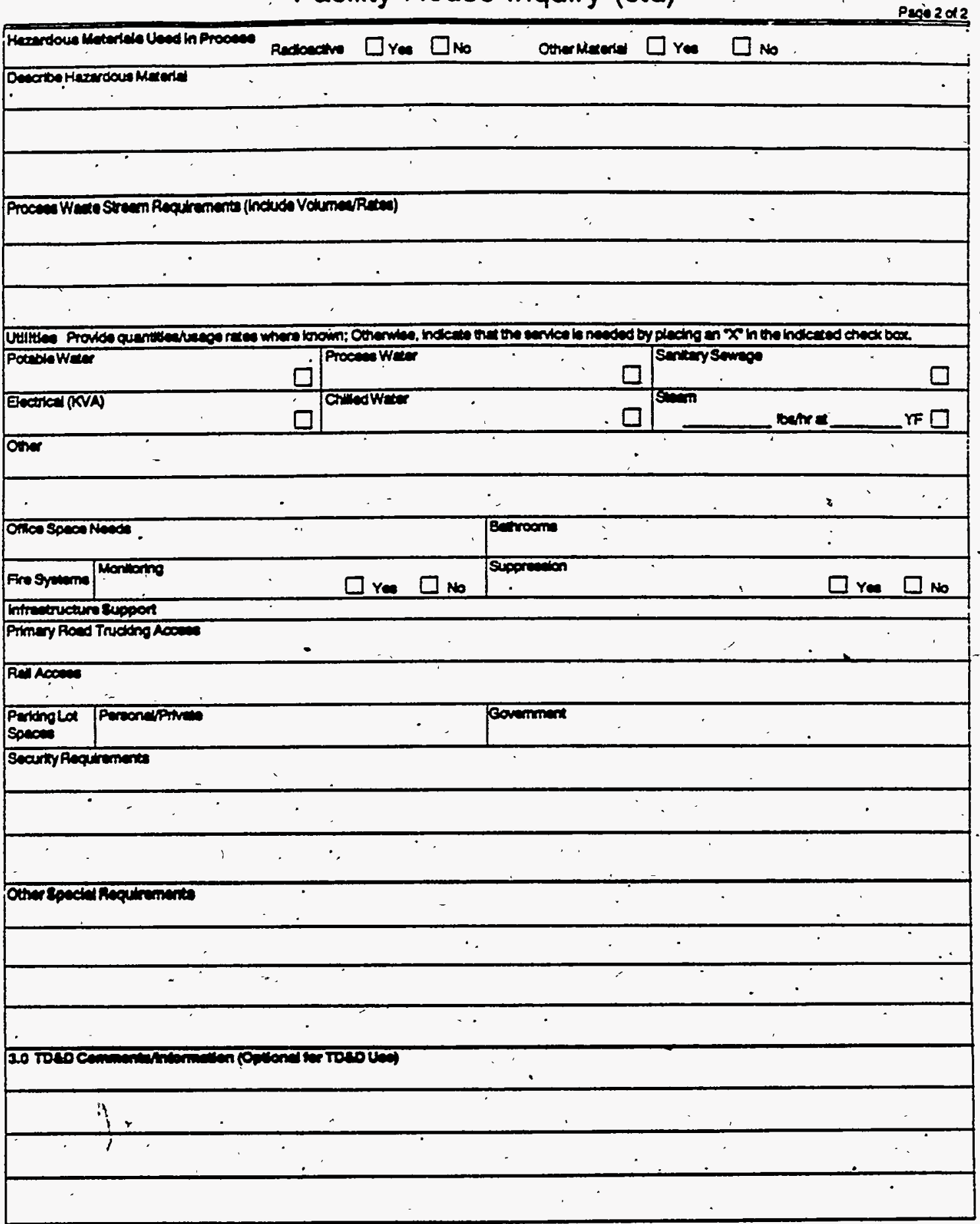

Appendix C

Facility Reuse Inquiry Form (back of form) 


\section{Distribution:}

N. C. Boyter, 991-W

F. B. Davis, 703-A

P. D. Grefenstette, 703-A

C. M. Hammond, 703-A

J. L. Hardin, 773-A

G. R. Harley, 703-A

H. A. Harmon, $719-4 \mathrm{~A}$

J. N. Herrmann, 703-A

G. K. Hovey, 991-W .

S. A. Johnson, 703-A

J. F. Jordan, 703-F

G. A. Krist, 703-A

R. Mahér, 703-A

I. B. New, 704-C

E. P. Rahe, 730-B'

A. B. Scott, 703-A

M. J. Sujka, 703-A

C. W: Thiessen, 703-A

J. G. Wise, 703-A

S. Wood, 773-A

J. G. Erwin, USFS, 760-G

J. D. Strawbridge, USFS, 760-1G

R. I. Nestor, SREL, 737-A

L. Brede Jr., WSI, 703-B

J. C. Noah, 773-A

T. R. Neal, 704-C

J. R. Yanek, 704-C

G. H. Clare, 704-C

C. H. Giuntini; $966 \mathrm{~W}$

G. F. Jernigan, 773-41A

M. V. Eidson, 999-W

R. H. Finley, 707-C.

D. W. Lentz, 707-C

M. B. Owen, 707-C

M. A. Brins $707 \mathrm{C}$

R. M. Clarke, 707-C

C. W. Van Horn, DOE, 703-46A

C. A. Everatt, DOE, 704-K

M. F. Salazar, DOE, 704-K

R. D. Jernigan DOE, 730-B

T. C. Walker, DOE, 703A

M: James, DOE, 703-46A

File $1010,707-\mathrm{C}$ 